\title{
AMBIENTES DE EDUCAÇÃO À DISTÂNCIA PARA O ENSINO DE ENGENHARIA: PROPOSTA DE MAPA CONCEITUAL
}

\author{
Autimio Batista Guimaraes Filho - afilho@uneb.br \\ Universidade do Estado da Bahia-UNEB-BA, Departamento de Ciências Exatas e da Terra \\ Rua Adelino Santos, 47 Liberdade,
}

CEP 40375430 - Salvador - Bahia

Fausto Ferreira Costa Guimarães - fausto_guimaraes@hotmail.com

Universidade do Estado da Bahia-UNEB-BA, Pró-reitoria de Infraestrutura

Rua Bahia, 615, apt 301, pituba,

CEP 41830-161, Salvador-Bahia.

Lila Martins Guimaraes - lila.guimaraes@ucsal.edu.br

Universidade Católica do Salvador-BA, Escola de Engenharia Civil

Travessa Sítio do Pombal, 30, Pituaçu

CEP 41741420 - Salvador - Bahia

Resumo: Estamos numa intensa fase de desenvolvimento de ideias criativas com ferramentas de Tecnologias de Informação e Comunicação (TICs) que projetam novos Ambientes de ensinolaprendizagem. Com a pandemia do COVID19, a Educação à Distância (EAD) passou a ser a solução diante da necessidade do afastamento social e muitas Instituições de Ensino Superior (IES) passaram a usar meios diversos, em todo mundo. Assim, este artigo, apresenta parte dos estudos para uma tese de doutorado, que avaliou as funcionalidades que devem possuir as ferramentas presentes nos Ambientes para suportar o ensino/aprendizagem, inclusive, de projeto de Engenharia, Arquitetura e Construção Civil. Para tanto teve como problema de pesquisa: os Ambientes de Educação à distância apresentam ferramentas com funcionalidades capazes de suportar as ações de projeto colaborativo na área de Engenharia, Arquitetura e Construção Civil? Com este estudo chegou-se a um mapa conceitual de um Ambiente educacional que apresenta ferramentas com funcionalidades necessárias para o ensino/aprendizagem, inclusive de disciplinas desta área. Espera-se contribuir com a melhoria do ensino sobre tudo à distância na área da Engenharia.

Palavras-chave: Educação à Distância, Ambientes de Educação, mapa conceitual, projeto colaborativo. 


\section{INTRODUÇÃO}

Estamos, na realidade, numa intensa fase de desenvolvimento de ideias criativas com ferramentas de Tecnologias de Informação e Comunicação (TICs) que projetam novos Ambientes de ensino/aprendizagem, novas rotinas e métodos pedagógicos que favorecem o desenvolvimento da colaboração eficiente dentro e entre universidades. Com a pandemia do coronavírus, a EAD passou a ser a solução diante da necessidade do afastamento social e muitas Instituições de Ensino Superior (IES) passaram a usar meios diversos para atender a continuidade da educação em todo mundo.

A Educação à Distância $(\mathrm{EaD})$ apoiada pelas tecnologias computacionais representa um novo cenário, onde a palavra colaboração passa a ter importante significado nas estratégias do ensino/aprendizagem e as dinâmicas colaborativas apresentam-se, então, como importantes componentes dessas estratégias educativas para a formação profissional. Nesta modadlidade de educação os Ambientes de Educação à Distância têm como objetivo facilitar o processo de oferecer cursos pela internet ainda que o professor não seja um especialista em computação ou em tecnologia computacionais para elaborar, ministrar e acompanhar o desenvolvimento de seus alunos. No entanto, professores e alunos continuam tendo dificuldades no uso dessas tecnologias com ferramentas ausentes ou inadequadas.

Diante do exposto, este trabalho assumiu como questão principal: os Ambientes de Educação à distância apresentam ferramentas com funcionalidades capazes de suportar o ensino/aprendizagem, inclusive, de projeto de Engenharia, Arquitetura e Construção?

Partiu-se da hipótese, à luz de autores que estudaram o assunto, de que os Ambientes de Educação à distância não apresentam ferramentas com funcionalidades capazes de suportar as ações de projeto colaborativo na área de AEC, servindo, principalmente, como repositório dos arquivos utilizados nas tarefas.

Para realizar este estudo, inicialmente, parte de uma pesquisa de doutorado, utilizou-se como metodologia a análise de Ambientes colaborativos, tanto para projetos de AEC, como, Ambientes de Educação à Distãncia, com o objetivo de verificar se as funcionalidades das ferramentas neles existentes, seriam capaz de suportar a educação em AEC, principalmente.

Assim, neste artigo, diante da crescente utilização de meios diversos para atender o ensino/aprendizagem devido a pandemia do COVID19 e preocupado com a qualidade da educação à distância, que certamente será sempre crescente, tem-se como objetivo apresentar um mapa conceitual, de um ambiente de EAD que poderá servir de diretrizes ou modelo

Espera-se contribuir com a educação em AEC, principalmente, visto que nos cursos nesta área, em particular, encontra-se disciplinas que exigem maior controle no processo de ensino/aprendizagem, possibilitando ao aluno maior entendimento daquilo que lhe está sendo apresentado e que certamente fará diferença no desenvolvimento do seu curso e ainda na sua atuação profissional futura.

\section{REFERENCIAL TEÓRICO}

Para o aprofundamento teórico da questão e desenvolvimento do estudo, procurou-se autores que trataram da educação à distância, no que diz respeito às funcionalidades das ferramentas que devem estar presentes nos ambientes de Educação à distância e ainda autores que apontaram preocupação com a necessidade urgente da utilização de ferramentas educacionais devido a pandemia do COVID 19. 
(C) COBENCE

"Os desafios para formar hoje o engenheiro do amanhã"

\subsection{Educação à Distância diante da pandemia do COVID 19}

Segundo Guimarães Filho (2010) apud Nunes (1994), Educação à Distância pressupõe um processo educativo sistemático e organizado que exige, não somente a dupla via de comunicação, mas também, a instauração de um processo continuado em que os meios ou multimeios devem estar presentes na estratégia de comunicação. Ainda, para o autor, a escolha de determinado meio ou multimeios vem em razão do tipo de público, custos operacionais, eficácia para a transmissão, recepção, transformação e criação do processo educativo. Complementando Boaventura (2020) aponta que a Educação a Distância é uma modalidade no Brasil, regulamentada pelo ministério de Educação e Cultura - MEC, onde há interação entre docentes e discentes que requer inserção da tecnologia digital da Informação e Comunicação (TDIC), pessoal qualificado, avaliações compatíveis, projeto pedagógico próprio e superação espaço-temporal.

Para Sandars et al (2020), a pandemia do COVID-19 resultou em uma súbita dependência do uso do aprendizado on-line o que requer atenção cuidadosa para garantir que haja otimização dos recursos disponíveis. Fazem-se necessárias mudanças efetivas para adaptar as tecnologias disponíveis e garantir a participação colaborativa de professores e alunos durante todo o processo. Além disso deve-se consider sustentar o uso do aprendizado on-line após a pandemia, em vez de simplesmente voltar à rotina tradicional de ensino presencial. Complementando, a Universidade Nacional Autônoma do México (UNAM) aponta 16 recomendações para a transição para o ensino presencial num documento dirigido aos professores e à comunidade acadêmica em geral, que enfrentam o complexo processo de educação presencial e não presencial, dada a atual contingência, como resultado da pandemia de COVID 19.

\subsection{Ambientes de Educação à Distância}

Autores como, Victorino e Haguenauer (2004) e Bassani (2006), refere-se aos Ambientes de Educação ou Ambientes de Educação à Distância com nomenclaturas diversas: Ambientes Computacionais; Ambientes de Colaboração na Educação ou Sistemas de Gerenciamento de Cursos; Ambientes de Aprendizagem à Distância, Ambientes Virtuais de Aprendizagem, Ambientes Colaborativos de Aprendizagem, dentre outros. Neste artigo será utilizada a denominação de Ambientes de Educação à Distância, sendo estes também considerados Ambientes Colaborativos. Estes Ambientes são aplicações da internet executados em servidores por um navegador $w e b$, localizados em qualquer parte do mundo, normalmente em centros de processamentos de uma Universidade acessados por professores e alunos de qualquer lugar com um computador conectado à internet.

Victorino e Haguenauer (2004) declinam que o Ambiente de Educação à Distância é um espaço de interação que sustenta a construção, inserção e troca de informações pelos participantes de um curso, visando à construção social do conhecimento. Segundo Bassani (2006), estes Ambientes apresentam um conjunto de características em comum: permitir acesso restrito a usuários previamente cadastrados, disponibilizam espaço para a publicação de material do professor, espaço para armazenamento de tarefas realizadas pelos alunos, e, ferramentas de comunicação síncrona e assíncrona. Ainda para Bassani (2006) os ambientes permitem a centralização de todas as informações referentes a um curso, porém o gerenciamento destas informações fica sob a responsabilidade de cada participante. $\mathrm{O}$ professor, neste contexto, percebe-se diante de um emaranhado de informações diluídas entre as várias ferramentas.

Segundo Victorino e Haguenauer (2004) o Ambiente de Educação à Distância surgiu a partir da prática do que se denomina Trabalho Cooperativo Apoiado por Computador (Computer Supported Cooperative Work - CSCW) criado por Geif e Cashman, em 1984. 
Posteriormente, a expressão Aprendizagem Cooperativa Auxiliada por Computador (Computer Supported Cooperative Learning - CSCL) vem sendo utilizada para designar uma abordagem em que o computador é utilizado como uma ferramenta que facilita a aprendizagem.

\subsection{Ferramentas dos Ambientes de Educação à distância e suas funcionaçidades}

Nestes Ambientes, permitindo as relações em que os estudantes possam refletir colaborativamente no interrelacionamento entre trabalhos e ideia, algumas ferramentas exigem sincronicidade de seus participantes, ou seja, que todos estejam conectados ao mesmo tempo tais como o bate-papo, por exemplo. Outras ferramentas permitem que cada um acesse assincronamente, como os fóruns, e-mails, blogs, dentre outros. Cada ferramenta se encaixa a diferentes materiais e objetivos, bem como se adéqua melhor à diferente tipo de usuário.

Diz Caldeira (2004) que, a assincronicidade e a independência de lugar para realização do curso, fazem da Educação à Distância uma forma mais flexível e adaptada às condições dos estudantes, mas, por sua vez, exigem maior disciplina. Essas características pressupõem o trabalho com alunos mais ativos em relação à própria aprendizagem, exigindo o desenvolvimento dessa habilidade ao longo do processo.

Ainda segundo este autor, essas formas diversificadas de interação, a possibilidade do registro dessas interações, as formas de intervenção do professor e dos pares são algumas das características que configuram os Ambientes Colaborativos de aprendizagem como espaços totalmente diferenciados dos presenciais. Complementando Guimarães Filho (2010) aponta que estes Ambientes devem, portanto, apresentar ferramentas capazes de possibilitar que o professor disponibilize uma série de materiais didáticos, aplique técnicas de dinâmicas de trabalhos individuais e de grupo e possibilite que os alunos, por sua vez, executem as tarefas propostas. Ademais, é fundamental que estes Ambientes apóiem o trabalho interativo, de forma que os alunos possam organizar e compartilhar ideias para que todos construam o seu conhecimento de maneira interativa e colaborativa e ainda que estes Ambientes possam registrar a participação dos alunos na execução das tarefas.

\subsection{A avaliação do aprendizado na Educação à Distância}

Aponta Guimarães Filho (2010) que a avaliação da colaboração bem como do aprendizado constitui sempre um problema na Educação à Distância. No entanto, de acordo com o T\&L. National Forum For the enhancement of teaching and learning in higher education, embora em alguns casos possa não haver uma alternativa on-line viável para avaliações com práticas baseadas em laboratório devido à necessidade de equipamentos especializados, alternativas podem ser possíveis:

- Simulações on-line podem ser usadas para permitir que os alunos aprendam e sejam avaliados com base em conhecimentos práticos, por exemplo, com os alunos tendo acesso a conjuntos de dados que são solicitados a interpretar.

- Também pode ser possível para os alunos fazer upload de gravações de vídeo realizando uma tarefa prática, se essa tarefa puder ser replicada com segurança e de maneira apropriada em sua casa.

- As gravações em áudio podem ser usadas para substituir avaliações orais,

- As entrevistas / exames orais podem ser realizados on-line usando as opções de videoconferência. Ao usar a videoconferência para avaliação, será importante garantir que a gravação da avaliação seja mantida, caso seja necessário para examinadores externos. Os alunos também precisarão estar cientes de que a entrevista / exame está sendo gravada. 


\section{DESENVOLVIMENTO}

Diante da real necessidade de utilização de Ambientes de Educação à Distância neste momento de pandemia do COVID 19, para dar continuidade às atividades educacionais e principalmente sua provável crescente utilização após a pandemia, fica evidente observar a qualidade dos ambientes a serem utilizados. Após análise de ambientes de trabalhos colaborativos e ainda ambientes de Educação à Distância foi possível observar a carência de ferramentas, bem como funcionalidades deficientes de ferramentas existentes que possibilitem o processo de ensino/aprendizagem de qualidade, principalmente nos cursos de AEC, objeto do estudo. Assim, chegou-se diante das necessidades básicas, nestes cursos a proposta de ferramentas com funcionalidades capazes de atender essas demandas

\subsection{Proposta de mapa conceitual de Ambiente para ensino/aprendizagem, de projeto de engenharia Arquitetura e Construção}

Segundo Guimarães Filho (2010), as ferramentas com funcionalidades que possibilitam a colaboração em projeto de AEC tais como ferramentas para comunicação síncrona e assíncrona como o bate-papo e fórum de discussão; editores colaborativos, como a wiki e ferramentas que possibilitam compartilhamento de arquivos diversos, dentre outros, são fundamentais para o trabalho e ensino/aprendizagem colaborativa. Ferramentas Apoio e Administração do Ambiente, Cronograma, Perfil, e ferramentas que possibilitam acompanhar a atuação dos dos alunos, são também necessárias. Ainda para o autor muitas destas ferramentas já estão presentes em Ambientes de Ensino à Distância, no entanto, fazem-se necessárias melhorias e ou ampliação das suas funcionalides, incorporar outras que possibilitam o acompanhamento da participação dos alunos, o que irá auxiliar o professor na condução das disciplinas e ainda na avaliação final.

A partir das propostas de Nitithamyong e Skibniewski (2004), dentre outros e das análises observou-se que um Ambiente de Ensino à Distância, para possibilitar a colaboração em projeto de AEC, necessita, fundamentalmente, de algumas seções com ferramentas que suportem tais funcionalidades, quais sejam: seções com ferramentas de suporte e administração; seções com ferramentas de apresentação da disciplina; seções com ferramentas que promovam a interação e colaboração através da comunicação; seções com ferramentas de avaliação; seções com ferramentas de configurações. O Quadro 1 mostra o resumo das seções com as ferramentas sugeridas para um Ambiente de Ensino à Distância.

Quadro 1 Sugestões de seções e ferramentas

\begin{tabular}{|l|l|}
\hline Seções & Ferramenta Sugerida \\
\hline Sobre o curso* & Dinâmica do Curso*; cronograma* \\
\hline Conteúdo* & Aulas*; Material de apoio*; Atividades* Portfólio**. \\
\hline Comunicação** & $\begin{array}{l}\text { Correio**; Fórum**; Laboratório Remoto*; Comunicador } \\
\text { Instantâneo**; Mural**; Enquete*; Editor colaborativo*; } \\
\text { Prancheta (Whiteboard)**; Blogger**; Grupos**; } \\
\text { Agendamento** }\end{array}$ \\
\hline Avaliação* & Exercícios*; Avaliações*; O que você acha?*Participação \\
\hline Configurações** & Dados Pessoais**; Idioma**; Cores da interface** \\
\hline $\begin{array}{l}\text { Gerenciamento*(visualização } \\
\text { apenas pelo formador) }\end{array}$ & $\begin{array}{l}\text { Gerenciador de usuários*; Gerenciador de ferramentas* } \\
\text { Dados do curso*; Relatórios*; Suporte Técnico** }\end{array}$ \\
\hline
\end{tabular}

Fonte: Pesquisa direta

LEGENDA: * Possibilidade de edição somente pelo formador; ** Possibilidade de edição pelo formador e pelo aluno. 
Observa-se, no entanto, que outras seções de ferramentas podem se fazer necessárias a depender da proposta do curso como, por exemplo: sistema de busca interno que poderá se constituir numa maneira rápida e prática do usuário procurar por textos, vídeos, fotos, usuários, etc.

$\mathrm{Na}$ seção sobre o curso, as ferramentas, podem ser visualizadas por todos, porém, alimentadas somente pelos formadores. Nesta seção, o aluno poderá obter informações do curso por meio de ferramentas, como Cronograma por exemplo:

- A ferramenta de Dinâmica do curso contém informações sobre a estratégia pedagógica, técnicas de trabalhos de grupos a organização geral do curso;

- A ferramenta de Cronograma traz a programação de um determinado período da disciplina (diária, semanal, mensal, etc.) com a tarefa, local, data e tempo de duração e links para as atividades associadas.

A seção de Conteúdo deve possuir ferramentas visualizadas por todos, porém, algumas destas devem ser alimentadas somente pelos formadores para edição de aulas: documentos, textos, atividades diversas, slides, vídeos-aula, etc. Nesta seção, os formadores poderão disponibilizar:

- Aulas (material básico, essencial, para que o aluno compreenda o assunto e acompanhe o curso: leituras básicas, vídeos, slides);

- Material de apoio (material complementar, essencial para acompanhamento do curso como leituras básicas, vídeos, slides, dentre outros);

- Atividades (trabalhos e experimentos a serem realizados pelos alunos);

- Portfólio. Também nesta seção deve existir ferramentas para que alunos compartilhem depositem conteúdos que possam ou não ser compartilhados. É interessante acrescentar a esta ferramenta o recurso de controle de versões, de visualização do conteúdo gráfico, de markup e de discussão.

A seção de Comunicação deve possuir ferramentas de interação e colaboração: correio, fórum, laboratório de acesso remoto, comunicador instantâneo, mural, enquete, blogger, grupos, editor colaborativo, prancheta e agendamento:

- A ferramenta Correio é amplamente conhecida, sendo um sistema de correio eletrônico interno ao ambiente que permita cópia do e-mail para sistemas externos;

- A ferramenta Fórum permite a discussão em tópicos. O usuário deve ter a possibilidade de anexar arquivos;

- A ferramenta de Laboratório de Acesso Remoto permite desenvolver experimentos remotamente;

- A ferramenta Comunicador Instantâneo viabiliza a conversa em tempo real entre os participantes da disciplina com opção de ser somente em texto, por áudio e ainda por vídeo. Deve permitir o compartilhamento de slides e ou imagens. Deve permitir a desktop conferência para discussões e apresentações online com possibilidade de manipulação por todos simultaneamente, permitir comentários tipo caneta (edição). Além disso, deve possibilitar diálogos em reservados. Cada participante ao conectar-se ao Ambiente poderá escolher sua disponibilidade: disponível, ocupado, indisponível e invisível;

- A ferramenta Mural disponibiliza informações sobre assuntos extra-aulas: informações gerais, encontros e notícias, deixando o Ambiente mais descontraído e recreativo;

- A ferramenta Enquete permite realizar pesquisa de opinião, sondagem, com visualização gráfica e percentual do resultado;

- A ferramenta Blogger permite que os participantes se expressem, publiquem os seus trabalhos e vejam os trabalhos publicados dos colegas. Deve permitir armazenar 
arquivos individuais utilizados e/ou desenvolvidos durante o curso, compartilhar álbuns de fotos, vídeos e textos, permitindo comentários;

- A ferramenta Grupos esta ferramenta permite a criação de grupos para facilitar a distribuição e/ou desenvolvimento de tarefas. Pode ser feita tanto por alunos ou formadores Armazenar arquivos do grupo, com possibilidade de compartilhamento entre grupos. Observa-se que as atividades em grupo constituem o cerne da formação atual, tanto presencial quanto à distância já que as dinâmicas colaborativas envolve a interrelação de aspectos cognitivo/afetivos individuais e construção social do conhecimento, ocorrendo a identificação pessoal por meio da interação com outras pessoas;

- A ferramenta de Editor colaborativo possibilita contribuições na execução de textos e planilhas. Se necessário, deve conter ferramentas que possibilite visualizar e editar desenhos ou projetos de Engenharia, Arquitetura e Construção Civil. Deverá abrir e salvar arquivos de extensão $D W G, D W F, P P T, D O C$, TXT, HTML, dentre outras, como também, em $P D F$;

- A ferramenta Prancheta (Whiteboard) possibilita a colaboração na execução de um desenho. Deverá ler arquivos $D W G, D W F, P L T$ (entre outros formatos) para revisão com markups. Deve estar conectado com comunicador instantâneo para possibilitar discussões por áudio e vídeo enquanto trabalham;

- A ferramenta de Agendamento permite agendar horários individuais para a realização de atividades, tais como: experimentos de Acesso Remoto, monitorias, etc.

A seção de Avaliação possui ferramentas que possibilitam acompanhamento e mensuração das participações e aprendizado, e assim, a avaliação dos alunos feita pelo professor e a avaliação do curso feita pelos alunos. Pode ser composta pelas ferramentas de: exercícios, "o que você acha?" e participação:

- A ferramenta de Exercícios disponibiliza testes que possibilitem a construção e a verificação do aprendizado. Deve possibilitar que o formador construa questões objetivas e subjetivas e retornar os resultados aos alunos;

- A ferramenta "O que você acha?" permite que o aluno emita impressões pessoais a respeito de assuntos da disciplina, por exemplo, possibilitando que o professor conheça as necessidades e anseios dos alunos e tome medidas mitigadoras, se necessário. É imprescindível que esta ferramenta possibilite o anonimato dos alunos, para que possam se expressar livremente;

- A ferramenta de Participação permite o acompanhamento da participação em tempo real, tanto pelos alunos quanto pelos formadores durante as atividades síncronas e assíncronas, tipo bate-papo, fórum de discussão e edição colaborativa, etc.

A seção de Configurações deve possuir ferramentas para configuração do Ambiente e pessoal. Deve permitir alterar dados pessoais (Perfil), Alterar senha, Alterar login, selecionar Idioma da interface e selecionar cores em padrões pré-configurados.

A seção de Gerenciamento deverá possuir ferramentas para gerenciamento do Ambiente e da disciplina por meio de gerenciadores de usuário, ferramentas, dados da disciplina, relatórios e suporte técnico:

- A ferramenta de Relatórios possibilita visualizar o registro de uso do sistema por meio de mapas de interação, acessos, páginas mais acessadas, navegação (caminho, duração e profundidade da seção, última página visitada.

Cabe reiterar que, a visualização da sua própria participação e da participação dos colegas e grupos, pode incentivar a atuação dos alunos na realização das tarefas. Esta visualização feita pelo professor pode auxiliar no acompanhamento da colaboração entre os alunos. Observa-se também no triângulo vermelho as ferramentas que possibilitam a interação e 
(C) COBENGE

"Os desafios para formar hoje o engenheiro do amanhã"
$\mathrm{Ol} \mathrm{a} \mathrm{O3}$ de dezembro Evento On-line

efetiva colaboração na construção do conhecimento e, ainda, no retângulo vermelho, as ferramentas que permitem ao professor apresentar detalhadamente a estratégia pedagógica, bem como, a(s) técnica(s) de trabalhos, inclusive de grupos, que serão utilizadas na disciplina.

Portanto, observa-se que um Ambiente de Educação à Distância, sobretudo para processo de projeto de AEC, deve apresentar um mapa conceitual como mostra a Figura 1. Dá-se em destaque, no círculo vermelho, a ferramenta de participação. Esta ferramenta seria para uso conjunto com uma ferramenta de discussão síncrona tipo Bate-papo. A ampliação da funcionalidade desta ferramenta poderia torná-la capaz de visualizar a participação, também, da atuação dos alunos ou grupos de alunos nas atividades executadas com as ferramentas Fórum de Discussão e Editor Colaborativo.

Figura 1 Mapa conceitual de Ambiente de Educação à Distância para AEC proposto.

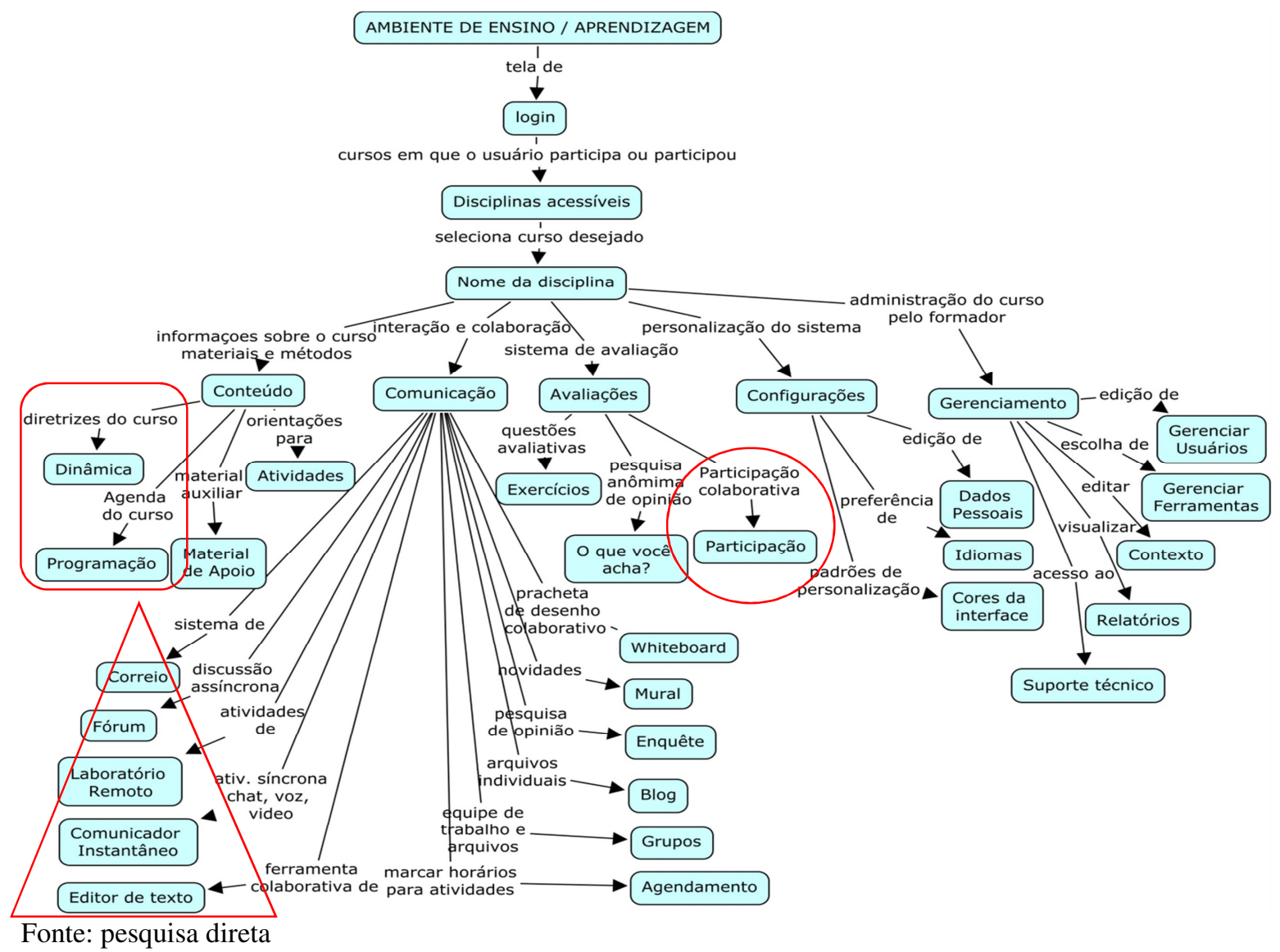

\section{CONSIDERAÇÕES FINAIS}

Não se pode negar a importância da qualidade do Ambiente de Educação no sucesso de um curso online, no entanto observa-se que apenas as ferramentas computacionais não são suficientes para promover de maneira eficiente a colaboração entre os membros de grupos nessa modalidade de ensino/aprendizagem. Deve-se dar maior importância, também, ao aspecto pedagógico, assegurar a construção do conhecimento de maneira colaborativa e nao utiliazar o ambiente apenas como meio de transmissão de informações ou simplesmente repositório. Além disso, deve-se dar atenção às atuações dos alunos, acompanhando as interações entre eles com ferramentas, presentes nos Ambientes de EaD. 
Observa-se que diferentemente dos Ambientes Colaborativos utilizados no campo profissional aqueles utilizados na educação, precisam apresentar ferramentas com funcionalidades mais amplas, para atender as dinâmicas aplicadas nas disciplinas e também, o acompanhamento da colaboração de maneira facilitada por parte do professor, para não só avaliar sua disciplina como também os alunos.

Devido ao fato do aprendizado colaborativo acontecer por meio do compartilhamento de informações na construção coletiva do conhecimento, acompanhar a atuação dos alunos com mensuração da colaboração é de fundamental importância para o professor. Ferramentas com funcionalidades que permitam a visualização da existência, ou não, de interação entre os participantes, como Fórum, Bate-papo, Wiki e Correio, por exemplo, possibilitarão esse acompanhamento e mensuração. Finalmente cabe lembrar a importância do treinamento prévio dos usuários dos ambientes para o devido aproveitamento das suas ferramentas.

Espera-se, portanto, que os desenvolvedores de softwares e provedores de serviço, possam considerar na criação das ferramentas dos Ambientes de Educação à Distância, a possibilidade delas favorecerem a participação de maneira colaborativa durante a realização das atividades e que seja considerada a possibilidade do acompanhamento dessas atuações, tanto por parte dos alunos e principalmente dos professores. Ademais que as instituições de ensino levem em consideração a necessirade destas ferramentas com as funcionalidades propostas, neste mapa conceitual, no momento de escolherem o Ambiente educacional.

\section{REFERÊNCIAS}

BASSANI, Patrícia B. S. Mapeamento das interações em ambiente virtual de aprendizagem: uma possibilidade para avaliação em educação à distância. 2006. 181f. Tese (Doutorado) - Universidade Federal do Rio Grande do Sul, Porto Alegre.

BENEVIDES, T.M; BOAVENTURA, L.PIMENTA. Mediação tecnológica e o enfrentamento à Covid-19. Jornal A TARDE, Salvador-BA, 23.06.2020. Disponivel em: https://joserosafilho.wordpress.com/2020/06/24/mediacao-tecnologica-e-oenfrentamento-a-covid-19/.Acessado em 05/07/2020.

CALDEIRA, A. C. M. Avaliação da aprendizagem em meios digitais: novos contextos. In: CONGRESSO INTERNACIONAL DE EDUCAÇÃO A DISTÂNCIA, 11, 2004, Salvador. Anais ... Salvador: ABED, 2004. Disponível em: <http://www.abed.org.br/congresso 2004/por/htm/159-TC-D3.htm> Acesso em: 10 ago 2007.

CHRISTIANSEN, E. Educated by design - learning by doing - outline of a HCI-didactics. ITcon, v. 9, p. 209-217. Disponível em: <http://www.itcon.org/2004/14/> Acesso em: 15 mai 2004.

GUIMARÃES FILHO, Autimio Batista. Dinâmica da colaboração em ambientes de educação à distância. Campinas, São Paulo, 2010, 350 f. Tese (Doutorado em Engenharia Civil) - Faculdade de Engenharia Civil, Universidade Estadual de Campinas.

NITITHAMYONG, P.; SKIBNIEWSKI, M.J. Web-based construction project management systems: how to make them successful? Automation in Construction, v. 13, n. 4, p. 491506, July 2004. 
"Os desafios para formar hoje o engenheiro do amanhã"

SANDARS J, et al. Twelve tips for rapidly migrating to online learning during the COVID-19 pandemic. Disponivel em:

https://docs.google.com/document/d/14SbZWavgv7egyruh8YJE3ywKi8uB95Jnx_S6xgyO owM/edit. Acessado em 15 jun 2020.

UNIVERSIDAD NACIONAL AUTÓNOMA DE MÉXICO (UNAM). Recomendaciones para la transicion a la docencian nopresencial, abril 2020. Disponivel em https://www.codeic.unam.mx/wpcontent/uploads/2020/04/Recomendaciones_Evaluaci\%C3\%B3n_Educativa_a_distancia.pdfA cesso em 10 jun 2020

T\&L. National Forum For the enhancement of teaching and learning in higher education.

Selecting Online Alternatives to Common Assessment Methods Supporting those who Teach and Learn in the Context of Covid 19 Coronavirus. Disponivel em: https://www.teachingandlearning.ie/wp-content/uploads/Assessment-methods-resource-forpublication-final.pdf. Acessado em 5 jun 2020.

VICTORINO, A.L. Q; HAGUENAUER, C. J. Avaliação em EAD apoiada por Ambientes Colaborativos de Aprendizagem no programa de capacitação para a Qualidade da COPPE/UFRJ. In: CONGRESSO INTERNACIONAL DE EDUCAÇÃO A DISTÂNCIA, 11., 2004, Salvador. Anais... Salvador: ABED, 2004. Disponível em:

<http://www.abed.org.br/congresso2004/por/htm/159-TC-D3.htm> Acesso em: 10 mar 2020.

\title{
DISTANCE EDUCATION ENVIRONMENTS FOR ENGINEERING EDUCATION: CONCEPT MAP PROPOSAL
}

\begin{abstract}
We are in an intense phase of developing creative ideas with Information and Communication Technologies (ICT) tools that design new teaching / learning environments. With the pandemic of COVID19, Distance Education (EAD) became the solution in the face of the need for social withdrawal and many Higher Education Institutions (HEIs) started to use different means, all over the world. Thus, this article presents part of the studies for a doctoral thesis, which evaluated the functionalities that the tools present in the Environments must have to support teaching / learning, including engineering, architecture and civil construction projects. For that, it had as a research problem: do the Distance Education Environments present tools with functionalities capable of supporting collaborative design actions in the area of Engineering, Architecture and Civil Construction? With this study, a conceptual map of an educational environment was arrived at, which presents tools with functionalities necessary for teaching / learning, including disciplines in this area. It is expected to contribute to the improvement of teaching about everything at a distance in the area of Engineering.
\end{abstract}

Keywords: Distance Education; Education Environments; conceptual map; collaboration design 